
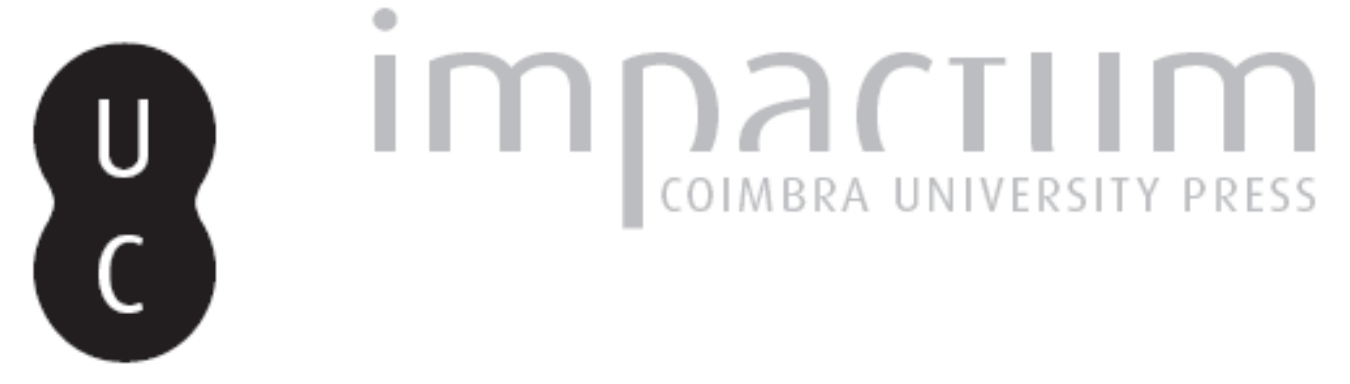

\title{
Burnout: um risco no desempenho e satisfação profissional nos bombeiros que trabalham na emergência pré-hospitalar
}

Autor(es): $\quad$ Vara, Natália; Queirós, Cristina

Publicado por: Associação Portuguesa de Riscos, Prevenção e Segurança

URL persistente:

URI:http://hdl.handle.net/10316.2/36166

DOI:

DOI:http://dx.doi.org/10.14195/1647-7723_16_17

Accessed : $\quad$ 26-Apr-2023 06:53:09

A navegação consulta e descarregamento dos títulos inseridos nas Bibliotecas Digitais UC Digitalis, UC Pombalina e UC Impactum, pressupõem a aceitação plena e sem reservas dos Termos e Condições de Uso destas Bibliotecas Digitais, disponíveis em https://digitalis.uc.pt/pt-pt/termos.

Conforme exposto nos referidos Termos e Condições de Uso, o descarregamento de títulos de acesso restrito requer uma licença válida de autorização devendo o utilizador aceder ao(s) documento(s) a partir de um endereço de IP da instituição detentora da supramencionada licença.

Ao utilizador é apenas permitido o descarregamento para uso pessoal, pelo que o emprego do(s) título(s) descarregado(s) para outro fim, designadamente comercial, carece de autorização do respetivo autor ou editor da obra.

Na medida em que todas as obras da UC Digitalis se encontram protegidas pelo Código do Direito de Autor e Direitos Conexos e demais legislação aplicável, toda a cópia, parcial ou total, deste documento, nos casos em que é legalmente admitida, deverá conter ou fazer-se acompanhar por este aviso.

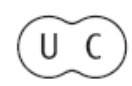




\section{territorium}

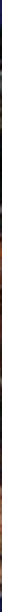

16

Revista da Associação Portuguesa de Riscos, Prevenção e Segurança 2009 


\section{BURNOUT - UM RISCO NO DESEMPENHO E SATISFAÇÃO PROFISSIONAL NOS BOMBEIROS QUE TRABALHAM NA EMERGÊNCIA PRÉ-HOSPITALAR*}

Natália Vara

Faculdade de Psicologia e de Ciências da Educação, Universidade do Porto vara.natalia@gmail.com

Cristina Queirós

Faculdade de Psicologia e de Ciências da Educação, Universidade do Porto cqueiros@fpce.up.pt

\section{RESUMO}

Procuramos conhecer o burnout e a satisfação profissional em bombeiros que trabalham na área da emergência pré-hospitalar, em diferentes zonas do país, tenob encontradb elevada satisfação profissional e reduzido bumaut, apesar do trabalho decorrer em situações emocionalmente intensas.

Palavras-chave: stress no trabalho, questionários, profissão de risco.

\section{ABSTRACT}

Bumat - a risk in the performance and job satisfaction in firefighters who works in emergency settings.

We tried to perceive bumaut and job satisfaction among firefighters who works in emergency settings, of different zones of portugal. We have found a higher job satisfaction and a lower level of bumaut, despite the professional activityocars instressful situations.

Key words: ocapational stress, survey, risk activities

\section{RÉSUMÉ}

Bumaut - un risque dans la performance et la satisfaction au travail des pompiers qui travaillent dans les situations d'urgence pré-hospitaliers

Nous voulons connaître le bumaut et la satisfaction professionnelle des pompiers qui travaille dans des contextes d'émergence, en différentes régions du Portugal. Nous avons trouvé beaucoup de satisfaction professionnel et peu laumat, même quand le travail est fait dans des conditions épuisantes.

Mbts-clé: stress dans le travail, questionnaires, professions de risque

\footnotetext{
* Commicação apresentada ao V Encontro Nacional e I Congresso Intemacional de Riscos.
} 


\section{Introdução}

Apesar de vivermos na chamada "sociedade do risco", o termo risco não se restringE à ideia de perigo e destruição que ocorre à volta do indivíduo. o contexto de trabal ho pode constituir um risco para a saúde do profissional que trabal ha em cenários de catástrofes, destruição, acontecimentos traumáticos e sofrimento. Segundo Pines (1993), aquele que tenta encontrar um significado na sua vida através do trabalho e sente que falhou, estará, provavelmente, mais exposto ao bumout. São aspectas relacionados com envolvimento, significação e motivação que podem ser determinantes para o risco desta síndrome. É uma experiência psicológica que implica sentimentos, atitudes e expectativas, sensação de esgotamento físico, mental e afectivo, atitudes frias para como autro, sensação de menor rendimento e inadequação no trabalho, afectandb o bem-estar geral da pessoa (I IrR et al., 1995) . O lamautéprincipalmente preditopelas exigências do trabalho, mas também pela falta de recursos no trabalho (SAryfHI \& Błkkr, 2004), desenvolvendo-se segundo um processo contínuo de desajuste entre as estratégias que o indivíduo usa para lidar como stress (estratégias de coping) e as exigências do trabalho (Gtrutss, 1980) . Os bombeiros são um exemplo de profissionais expostos a este tipo de situações, pois as ocorrências para as quais são chamados a intervir tomam formas diversificadas, não permitindo, muitas vezes, uma adaptação adequada. São por isso dorigados a lidar com pressão temporal, sobrecarga de responsabilidades e de horas de trabalho, exigências físicas, cognitivas e emocionais, rearsos por vezes limitados e expectativas sempre elevadas, o que afecta a satisfação profissional e a motivação (V̌RA, 2007) .

A natureza do trabalho desenvolvido pelos bombeiros que trabalham na área da emergência préhospitalar implica a necessidade de manter contacto directo com outras pessoas, sendo por excelência um contexto ande se pode menifestar o stress emocional, pois existe empenho, envolvimento, devoção a uma causa específica e, em conjugação, um trabalho sob "condições difíceis" . Estas condições podem residir na concepp̧ão e na gestão da organização do trabalho, bem como nas interacções entre trabalhadores e ambiente de trabalho. Ironicamente, o lam aut atinge precisamente os indivíduos mais ideal istas e entusiastas na sua profissão, aqueles que vão para a profissão com um forte desejo de se dar aos outros e sentem que realmente podem ajudar (PINES \& Aransan, 1989) .

De acordo com PINES e Mastach (1978), o burnout tem uma correlação negativa com a satisfação no trabalho, podendo ser visto como uma resposta emocional ao trabalho (BRENER \& CATPPARD, 2002).
CARNALO (1988) considera que existem perturbações de saúde que, apesar de não conduzirem à internupção da actividade nomal, aumentam o risco de acidentes, reduzem as potencialidades do indivíduo influenciam au perturbam a produtividade, as relações sociais e o equil 1̂́brio social e fami liar. Numerosos estudos relacionam o elevado burnout

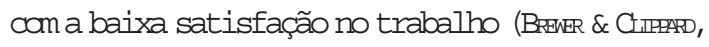
2002 ; HaLbeSLEBEN \& Bowler, 2007; MAsLACH, JACKSON \& LeITER, 1996; RAZZA, 1993). Alguns autores verificaram que a baixa satisfação no trabalho é a variável mais importante para predizer a exaustão profissional. O laumaut constitui por isso um risco, pois conduz à deterioração da saúde física e psíquica, a atitudes laborais alteradas que incidem directamente no desempenho profissional do indivícuo, reflectindo-se numa desumanização e deterioração na qualidade dos serviças prestados. São estas circunstâncias que nos permitem supor que a vulnerabilidade psíquica dos bombeiros que trabalham na área da emergência pré-hospitalar é semelhante à dos outros profissionais que actuam em catástrofes, mesmo que os constrangimentos e tarefas sejamdiferentes.

Tentamos com este estudo conhecer o burnout e a satisfação profissional em bonbeiros que trabal ham na área da emergência pré-hospitalar, bem como verificar se existem diferenças em função de variáveis profissionais e se existe uma correlação entre lam mat e satisfaçãoprofissional.

\section{Metodologia}

\section{Participantes}

Inquirimos 119 bombeiros tripulantes de ambulância de socorro, distribuídos de forma semelhante pelas três zonas do país (zona norte com 38 inquiridos, centro com 39 e sul com 42) , tendo uma média de idades de 31.84 anos ( $\mathrm{DP}=5.94)$. Predominou o sexo masculino (91\%) e o estado civil casado ou em união de facto (56\%). Relativamente à situação profissional, 47\% dos inquiridos são bombeiros voluntários assalariados que trabalham para uma Associação Humanitária, 29\% são bombeiros voluntários, 15\% são bombeiros municipais e 9\% são bombeiros sapadores. Trabal ham sobretudo por turnos (84\%) , com uma média de 44 horas semanais, tendo em média 10.4 anos de serviço na área da emergência pré-hospitalar.

\section{Material}

Questionário constituŕdo por três grupos de questões: caracterização socio-demográfica da 
amostra, avaliação da satisfação e motivação no trabalho (VARA, 2007) e avaliação do burnout (utilizando uma adaptação do Maslach Burnout Inventory, de Mastach \& Jacksov, 1997) .

\section{Procedimentos}

Os cadbs foram recolhidos em2006, durante os arrsos de re-certificaçãona Escolankacional deBonbeiros, sendb o questionário anónimo e de auto-preendhimento.

\section{Resultados}

A amostra apresenta maioritariamente um grau elevado de satisfação com o trabalho (65\%), concentrando-se nos graus 4 e 5 (numa escala de 1 a 5 pontos) e tendo uma média de 3.69 ( $\mathrm{DP}=0.89)$. Em relação à motivação para exercer o trabalho, 61\% das elementos da amostra apresentam um grau elevado, o que aliás se confi ma pela média dotida 3.69 (DP=0.98) . Quanto à motivação no início da profissão, verificamos uma preponderância das respostas para o grau elevado (84\%) , traduzida na média de 4.39 (DP=0.83) , respectivamente. Estes resultadbs são consistentes com as respostas sobre a satisfação e motivação com o trabalho. Verificamos, de ummodo geral, que a nossa amostra apresenta uma elevada satisfação no trabalho (média de 70.98 DP=11.05 para uma escala que pode variar de 19 a 95 pantos) , indicandb-nos que no geral os inquiridas estão bastantes satisfeitos com o seu trabalhoe, por conseguinte, não demonstram interesse emmudar de instituição au de funções. Estes resultadbs vêmao encontro de attros estudbs, pois os profissionais apresentam resultadbs significativamentemais elevadbs quando se sentem envolvidos no trabalho (interesse, variedade e tipo de tarefas realizadas) , quando têm boas relações com os colegas (e satisfação em trabalhar em equipa), apoio dos superiores, clareza nas suas tarefas e conforto físicono seu trabalho (Brick \& WoolcotT, 1996) .

Não foram encontradas diferenças significativas entre os dois sexos no que se refere à satisfação no trabalho. Quanto ao burnout, os dados permitiram concluir que os bombeiros do sexo masculino apresentam maior nível de despersonalização (Qtaro I) , o que vai de encontro aos resultados de

QuADRo I - Comparação das médias da satisfação com o trabalho e das dimensões do burnout em função do sexo

\begin{tabular}{|c|c|c|c|}
\cline { 2 - 4 } \multicolumn{1}{c|}{} & Feminino & Masculino & T student (p) \\
\hline Satisfação com o trabalho & 71,6 & 70,9 & $-0,2(0,838 \mathrm{NS})$ \\
\hline Exaustão emocional & 14,7 & 16,7 & $0,7(0,512 \mathrm{NS})$ \\
\hline Despersonalização & 3,5 & 6,7 & $2,9(0,008 * *)$ \\
\hline Realização pessoal & 38,6 & 39,1 & $0,2(0,858 \mathrm{NS})$ \\
\hline
\end{tabular}

já próxima do ponto máximo que é de 5, o que nos leva a concluir que a motivação no início da actividade profissional era mais acentuada. Os bombeiros que optam por trabal har nesta área procuram sobretudo aliar as funções desempenhadas a um ideal e dedicação a uma causa. Esta motivação prende-se com o facto de poderem trabal har com pessoas e prestar serviços de ajuda a essas pessoas (VARA, 2007) . contudo, tal como A. Vaz SerRA (1999) frisou, essa motivação diminui com o passar do tempo, se comprovam que não são apreciados.

Relativamente à possibilidade de mudar de funções naprofissão aude institurição, se tivessem poortunidade para isso, encontramos uma resposta negativa na maioria dos indivíduos, com 70\% e 79\% estudos (Mhsach et al., 2001) que apontam níveis de despersonal ização mais elevados no sexo mascul ino.

No que se refere à zona do país (Qसrro II) , não encontramos diferenças significativas no total de satisfação no trabalho (apresentando contudo as zonas norte e sul maior satisfação) . Contudo, encontramos diferenças significativas nos itens autonomia para tomar decisões e possibilidade de participar na tomada de decisão, sendo a zona norte a que evidencia maior grau de satisfação comparativamente às outras duas. Ao analisarmos a variedade de tarefas, constatamos a existência de valores estatisticamente significativos, apresentanobse os graus de satisfação mais elevados nas zonas norte e sul. Quanto à possibili idade de trabalhar em

QUADRo II - Comparação das médias da satisfação com o trabalho em função da zona do país

\begin{tabular}{|c|c|c|c|c|}
\cline { 2 - 5 } \multicolumn{1}{c|}{} & Norte & Centro & Sul & F (p) \\
\hline Satisfação com o trabalho & 73,0 & 68,2 & 71,8 & $1,9(0,161)$ \\
\hline Autonomia para tomar decisões & 3,7 & 3,1 & 3,3 & $3,8\left(0,025^{*}\right)$ \\
\hline Possibilidade de participar na tomada de decisões & 3,9 & 3,1 & 3,2 & $6,9\left(0,001^{* * *}\right)$ \\
\hline Variedade das tarefas profissionais & 4,1 & 3,6 & 4,0 & $3,3\left(0,040^{*}\right)$ \\
\hline Possibilidade de trabalhar em equipa & 4,1 & 3,9 & 4,4 & $3,3\left(0,042^{*}\right)$ \\
\hline Clima de relacionamento entre colegas & 4,2 & 3,9 & 4,3 & $3,6\left(0,031^{*}\right)$ \\
\hline
\end{tabular}

$* \mathrm{p}<0.05 * * \mathrm{p}<0.010$ 
RISCOS - Associação Portuguesa de Riscos, Prevenção e Segurança

QuADRo III - Comparação das médias do burnout em função da zona do país

\begin{tabular}{|c|c|c|c|c|}
\cline { 2 - 5 } \multicolumn{1}{c|}{} & Norte & Centro & Sul & F (p) \\
\hline Exaustão emocional & 16,8 & 19,8 & 13,3 & $4,9\left(0,009^{* *}\right)$ \\
\hline Despersonalização & 7,6 & 7,1 & 4,5 & $3,1\left(0,049^{*}\right)$ \\
\hline Realização pessoal & 39,6 & 37,1 & 40,3 & $2,1(0,124)$ \\
\hline
\end{tabular}

equipa, tanbém revelau diferenças significativas, pois o sul apresenta graus mais elevados de satisfação, logo seguido da zona norte. Por último, o clima de relacionamento entre os colegas evidenciou resultados estatisticamente significativos, sendo novamente as zonas do sul e norte que se destacam com as médias mais elevadas de satisfação. de trabalho (apresentandb respectivamente uma média de 3.0 e de 2.98 , contra 3.83 e 4.0 nos bombeiros municipais, $\mathrm{F}=3.5$ e $\mathrm{p}=0.018$, e $\mathrm{F}=5.1$ e $\mathrm{p}=0.002$ ) . Esta carga horária, bem como o horário de trabalho, surgem associados à exaustão emocional.

Encontramos ainda uma correlação negativa entre o total da satisfação com o trabalho e exaustão

QuñRo IV - Comparação das médias da satisfação com o trabalho e do bumout em função da situação profissional

\begin{tabular}{|c|c|c|c|c|c|}
\cline { 2 - 6 } \multicolumn{1}{c|}{} & Assalariado & Voluntário & Municipal & Sapador & F (p) \\
\hline Satisfação com o trabalho & 71,9 & 69,9 & 71,4 & 69,2 & $0,3(0,820)$ \\
\hline Exaustão emocional & 18,1 & 16,8 & 13,1 & 13,6 & $1,6(0,187)$ \\
\hline Despersonalização & 7,1 & 6,4 & 4,2 & 6,4 & $0,9(0,396)$ \\
\hline Realização pessoal & 40,6 & 36,8 & 40,1 & 36,5 & $2,4(0,068)$ \\
\hline
\end{tabular}

Para o laumat, as diferenças significativas siturmse na exaustão emocional e na despersonalização. A zona centro apresenta valores mais elevados ao nível da exaustão emocional, enquanto o sul apresenta valores mais elevados na despersonalização. Alguns estudas têm vindo a relacionar a exaustão emocional com as exigências do trabalho e a capacidade de controlo sobre a tarefa executada com a despersonalização, confirmando as consequências destas variáveis no desempenho profissional

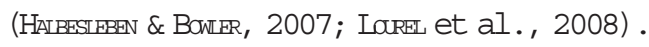

Ao compararmos as diferentes tipos de situação profissional dos bombeiros (QuAdRo IV), não emocional e uma correlação positiva entre o total da satisfação com o trabalho e a realização pessoal (Qurro V) . A motivação e a satisfação no trabalho apresentam correlações negativas com a exaustão emocional e correlações positivas com a realização pessoal. O número médio de horas semanais correlaciona-se positivamente com a exaustão emocional. Estes resultados são consistentes com vários estudos que comprovam a existência de uma correlação positiva entre exaustão emocional e amplitude do horário de trabalho (Mastad, ScFarferI \& LeTIER, 2001; Mindes, 2005; SPARKS et al. , 1997; Truchor, 2004).

QUADRO V - Correlação entre satisfação com o trabalho e bumout (R de Pearson e p)

\begin{tabular}{|c|c|c|c|}
\cline { 2 - 4 } \multicolumn{1}{c|}{} & $\begin{array}{c}\text { Exaustão } \\
\text { emocional }\end{array}$ & Despersonalização & $\begin{array}{c}\text { Realização } \\
\text { pessoal }\end{array}$ \\
\hline $\mathbf{N}^{\mathbf{0}}$ médio de horas semanais no trabalho & $0,247\left(0,008^{* *}\right)$ & $0,163(0,083)$ & $0,118(0,212)$ \\
\hline Satisfação no momento actual & $-0,284\left(0,002^{* *}\right)$ & $-0,086(0,355)$ & $0,208\left(0,023^{*}\right)$ \\
\hline Motivação no momento actual & $-0,381\left(0,000^{* *}\right)$ & $-0,178(0,053)$ & $0,302\left(0,001^{* *}\right)$ \\
\hline Total da satisfação com o trabalho & $-0,434\left(0,000^{* *}\right)$ & $-0,182(0,052)$ & $0,285\left(0,002^{* *}\right)$ \\
\hline
\end{tabular}

* $\mathrm{p}<0.05 * * \mathrm{p}<0.010$

encontramos diferenças significativas quanto à satisfação no trabal ho nem no laumout. No entanto, os bombeiros voluntários assalariados apresentaram mais satisfação com o trabalho e maior realização pessoal, mas também valores superiores de exaustão emocional e de despersonalização.

Uma análise mais detalhada da satisfação com o trabalho revel au que esta está sobretudb associada à carga horária das tarefas, tendb-se constatadb que os bombeiros voluntários assalariados estão menos satisfeitos coma carga horária das tarefas e o horário

\section{Conclusões}

Apesar da elevada satisfação profissional e do baixo bumout encontrados, os bombeiros também são vulneráveis ao bumout, atendendo ao facto de diariamente enfrentarem situações emocionalmente intensas, à necessidade de decidir muitas vezes sob pressão, com urgência e em condiçães de risco ou limitação de rearrsos. Verificamos que as bonbeiros do sexo masculino apresentam maior nível de 
despersonalização e que os bombeiros voluntários assalariados apresentam mais exaustão emocional, associada a menos satisfação com a carga horária das tarefas. Estes resultadbs são consistentes coma literatura, que indica uma associação entre exaustão emocional e sobrecarga de trabalho (GRFENGASS, Burke \& Firksenbalm, 2001) , podendo as exigências do trabalho predizer a despersonalização e a exaustão emocional (Lom et al., 2008) . Constatamos também que a zona centro do país apresenta maiores níveis de exaustão emocional enquanto as zonas norte e sul apresentammaior satisfação profissional. Foi também encontrada uma correlação negativa entre satisfação profissional e bumaut, que, tal como a literatura sugere, aparecem correlacionados com a fraca satisfação laboral (BREver \& C.TPPARD, 2002; HatbesteBen \& Bowler, 2007; Pines \& KeINan, 2005), podendo esta funcionar como um preditor da exaustão profissional (Napoes-TEXXERA, 2002) .

Para concluir, salientamos a importância de considerar a intensidade e o nível de exigência que esta actividade profissional requer, a necessidade de aumentar as fontes de motivação dos profissicnais, de garantir um clima benéfico de produtividade e de realização pessoal, com vista a prevenir o sofrimento psicológico e a promover a saúde e bem-estar dos bonbeiros, prestandb estes melhores aridadbs de saúde e não colocandb em risco as utentes a quem socorrem.

\section{Bibliografia}

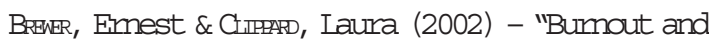
job satisfaction among student support services personnel" . Human Resource Development Quartely, vol.13 /2, San Francisco, p.169-186.

CARAaLH, Rui (1988) - "O médico do trabalho e o stress capacional". Psiquiatria Clínica, vol.9 / 4, São Paulo, p. 293-300.

Ctralss, Carry (1980) - Professional Bumout in the Hrman Service Organizations, Praeger, New York, $295 \mathrm{p}$.

Grenngass, Esther; Burke, Ronald \& Fiksendaim, Lisa (2001) - "Workload and burnout in nurses" . Joumal of Community and Applied Social Psychology, vol.11, New Jersey, p. 211-215.

HAIBESTHBEN, Jonathon \& BowerR, Matthew (2007) "Emotional exhaustion and job performance: the mediating role of motivation". Jamal of Applied Psychology, vol.92 / 1, Washington, p. 93-106.

Lror, Bartolomé; AвoD, Mateo; GARCTA, Mariano \& NIETO, Joaquin (1995) - "Fundamento psicosociales del compotamiento en las organizaciones" . Ciencias Psicosociales Aplicadas a la Salud, Interamericana MoGawHill, Madrid, p. 295-305.
Lourke, Marcel; AbDeifaor, Sid; CHevaleyre, Sandra; Paltrier, Maude \& Gana, Kamel (2008) "Relationship between psychological job demands, job control and burnout among firefihters". North American Jaumal of Psychology, vol. 10 / 3, Winter Garden (Ontário) , p. 489-496.

MFPQRE-JEXXEIRA, João (2002) - "Bumout ou a síndrome da exaustão". Saúde Mental, vol. IV / 2, Linda-aVellha, p. 8-19.

Masach, Christina; Jackson, Susan \& Leiter, Michael (1996) - Maslach Bumout Inventory Manual. Consulting Psychologists Press, Palo Alto, califómia, $52 \mathrm{p}$.

Mastach, Christina \& JAcrsan, Susan (1997) - MBI, inventário Bumout de Maslach, síndrome del "quemado" por estrés laboral asistencial mamal. TEA, Madrid, $36 \mathrm{p}$.

MkEAG, Christina \& I ENTER, Michael (1997) - The tnuth about bumaut: how organizations cause personal stress and what to do about it. Jossey-Bass Publishers, San Francisco, 182 p.

Mastact, Christina; Shatreri, Wilmar \& IeUTHR, Michael (2001) - "Job Burnout". Annual Review Psychology, vol.52, Califómia, p. 397-422.

Mindes, Miguel (2005) - Emoções no contexto de policiamento: medo, exaustão emocional e procura de sensações, um estudo comparativo na PSP do Porto. Dissertação de Mestrado em Criminologia, F.D.U.P. , Porto, 221 p.

PNes, Ayala \& Arosson, Elliot (1989) - Career bumaut, causes and arres. Collier Nacmillan Publishers, London, $257 \mathrm{p}$.

PRes, Ayala \& KENRN, Giora (2005) - "Stress andlarmat: thesignificant difference". PersonalityandIrodividal Differences, vol. 39, Amsterdam, p.625-635.

Pines, Ayala \& MASLACH, Christina (1978) "Characteristics of staff loumatt inmental health settings". Hospital and Commity Psychiatry, vol. 29 / 4, washington, p. 233-237.

RazzA, Nancy (1993) - "Determinants of direct-care staff tumover ingroup homes for individuls with mental retardation". Mental Retarcation, vol. 31, Washington, p. 284-291.

SCHAUFELI, Wilmar \& BAKKER, Arnold (2004) - "Job demands, job resarrces, and their relationship with bumout and engagement: a multi-sample study" . Joumal of Organizational Behavior, vol. 25, Hoboken, p. 293-315.

Shat, Adriano Vaz (1999) - O stress na vida de todos as dias. Gráfica de coimbra, Coimbra, 779 p.

Searks, Kate, CoOprr, Cary, Frited, Yitzhak \& SHrRaM, Arie (1997) - "The effects of hours of work on health: A meta-analytic review". Joumal of Occupational and Organizational Psychology, vol. 70, Leicester, p. 391-408. 
RISCOS - Associação Portuguesa de Riscos, Prevenção e Segurança

Thanr, Didier (2004) - Epouisement professionnel et lamat: Concepts, modèles, interventions. Dunod, Paris, $265 \mathrm{p}$.

VARA, Natália (2007) - Bumout e satisfação no trabalho em bombeiros que trabalham na área da emergência pré-hospitalar. Dissertação de Mestrado em Psicologia da Saúde. F.P.C.E.U.P., Porto, $200 \mathrm{p}$. 